Case Report

\title{
Susceptibility Weighted Imaging as a Useful Imaging Adjunct in Hemichorea Hyperglycaemia
}

\author{
Ferry Dharsono, Andrew Thompson, Jolandi van Heerden, and Andrew Cheung
}

Neurological Intervention and Imaging Service of Western Australia, Sir Charles Gairdner Hospital, Hospital Avenue, Nedlands, WA 6009, Australia

Correspondence should be addressed to Ferry Dharsono; ferry.dharsono@health.wa.gov.au

Received 15 April 2013; Accepted 9 May 2013

Academic Editors: C. Chaskis and R. Murthy

Copyright (c) 2013 Ferry Dharsono et al. This is an open access article distributed under the Creative Commons Attribution License, which permits unrestricted use, distribution, and reproduction in any medium, provided the original work is properly cited.

Hyperglycaemia with hemichorea (HGHC) is an unusual clinical entity that can be associated with corpus striatum hyperintensity on T1-weighted (T1W) magnetic resonance imaging (MRI) sequences. We report the utility of the susceptibility weighted image (SWI) sequence and the filtered phase SWI sequence in the imaging assessment of HGHC.

\section{Case Report}

A 54-year-old man presented to the emergency department with a 5-day history of right-sided HemiChorea on a background of type II diabetes and hypertension. There was no personal or family history of movement disorders and no history of neuroleptic drug use. At presentation, serum blood glucose level was $36.2 \mathrm{mmol} / \mathrm{L}$ with an $\mathrm{HbAlc}$ of $16 \%$. There was no ketonuria. The hemichorea resolved with optimal blood glucose control.

Initial nonenhanced CT (NECT) head demonstrated increased attenuation in the left corpus striatum, corresponding to hyperintensity on T1W MRI. There was no evidence of acute infarction on diffusion weighted imaging (DWI). The SWI sequence showed asymmetric hypointensity within the left lentiform nucleus, with sparing of the caudate nucleus (Figures $1(\mathrm{a})-1(\mathrm{~d})$ ). All MR studies were performed on a $1.5 \mathrm{~T}$ superconducting MR imaging system (Aera; Siemens, Erlangen, Germany).

Surveillance MRI at five months revealed volume loss of the entire left corpus striatum with partial resolution of the hyperintensity on TIW MRI, particularly at the caudate head. Small cystic foci of malacic change were demonstrated within the left posterolateral putamen. The SWI sequence revealed interval progression in the extent and degree of hypointensity in the left lentiform nucleus and caudate head. SWI hypointensity corresponded to areas of hyperintensity on the filtered phase SWI sequence (Figures $1(\mathrm{e})-1(\mathrm{~h})$ ). These findings are consistent with deposition of paramagnetic material in a left-handed coil MR system, such as the Aera (Siemens; Erlangen, Germany).

\section{Discussion}

Hemichorea is defined as continuous, nonrhythmic, involuntary movements affecting one side of the body. A focal vascular insult to the contralateral basal ganglia is considered to be the most common cause. Nonketotic hyperglycemia is a less usual cause for this presentation [1-3]. Affected patients are typically poorly controlled diabetics who experience acute-onset Hemichorea, hemiballismus, and occasionally, altered mental status [4], with imaging often demonstrating characteristic CT high attenuation of one or both corpus striati with associated abnormal hyperintensity on T1W MRI $[5,6]$.

The pathophysiological basis for striatal signal alteration in HGHC is unclear; however various hypotheses exist [4]. Some authors suggest microhaemorrhage as a cause [7], while other studies failed to demonstrate microhaemorrhage histopathologically, suggesting calcium deposition as the favoured aetiology [3]. Others consider cerebral ischemia as the most appropriate explanation of the $\mathrm{T} 1$ signal alteration $[8,9]$.

Histopathologically, Shan and coauthors reported the presence of abundant gemistocytes (reactive swollen astrocytes) without evidence of hemorrhage [3]. Ohara et al. 


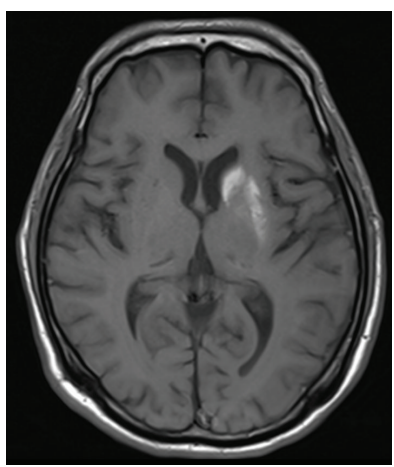

(a)

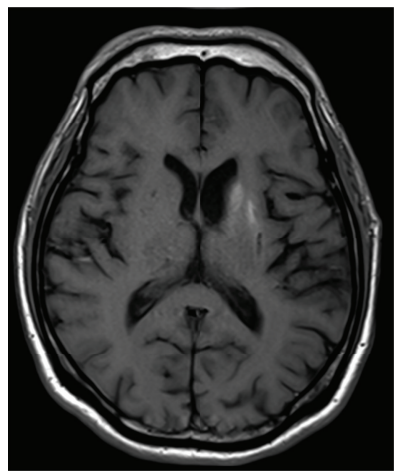

(e)

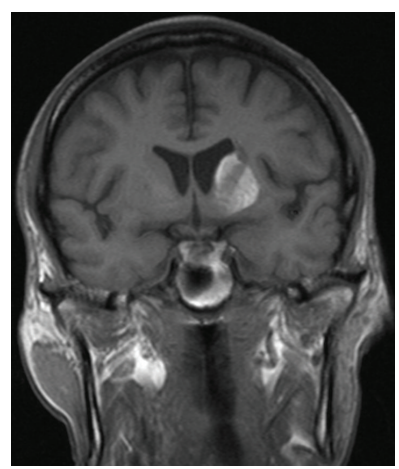

(b)

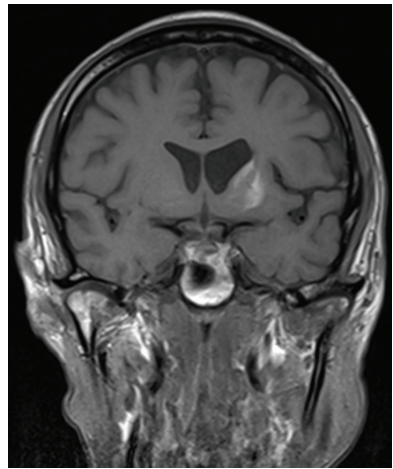

(f)

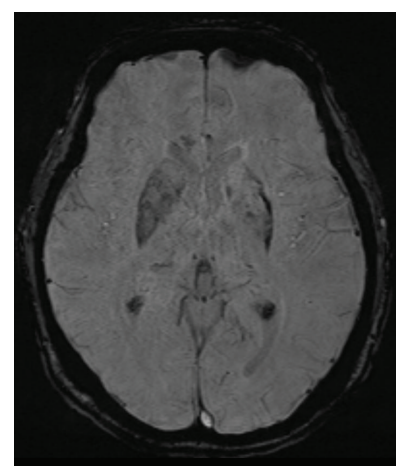

(c)

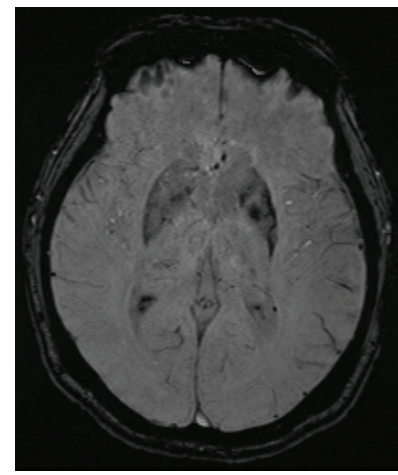

(g)

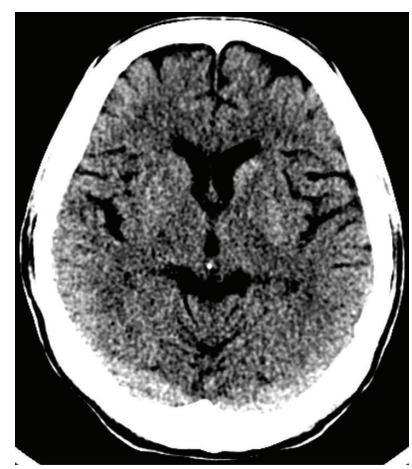

(d)

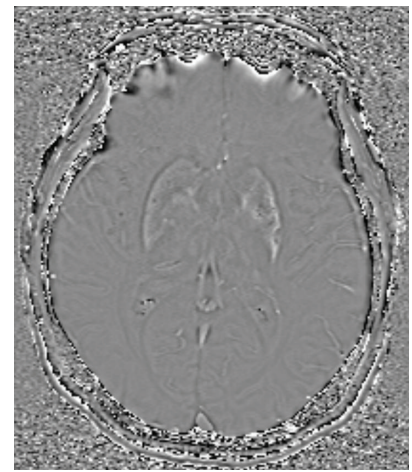

(h)

FIGURE 1: MRI changes in the left corpus striatum related to hemichorea hyperglycaemia. Top row: initial MRI head shows abnormal hyperintensity in the left caudate head and left putamen on axial and coronal T1W MRI (a) and (b). Asymmetric low signal was noted along the lateral margin of the left putamen on SWI (c). On NECT head (d) there is corresponding increased attenuation in the left corpus striatum. Bottom row: MRI head performed five months later shows volume loss of the entire left corpus striatum, cystic foci of malacic change in the posterolateral putamen along with partial resolution of the hyperintensity on axial and coronal T1W MRI (e) and (f). There was progression in extent and degree of hypointensity within the left corpus striatum on SWI (g) with corresponding hyperintensity on filtered phase SWI (h).

described autopsy-proven lacunar infarcts associated with reactive astrocytosis within the affected putamen [10] and Neal et al. showed mineral deposition in hypertrophied astrocytes located within ischaemic brain [11]. A case report by Cherian et al. suggests paramagnetic mineral deposition in the affected putamen caused by swollen gemistocytes that express metallothionein and zinc secondary to ischaemic insult [1]. The hypothesis of transient ischaemia was supported by Mittal who described the presence of ipsilateral prominent cortical veins on SWI sequences [12].

Our case report explores the utility of the final SWI and filtered phase SWI sequences in identifying the nature of the striatal hyperintensity.

SWI is a relatively recent MR technique that exploits susceptibility differences between tissues by using both magnitude and phase images from a high resolution $3 \mathrm{D}$ fully velocity compensated radio-frequency (RF) spoiled gradient echo sequence. The phase image, which detects differences between tissues, is corrected by applying a high pass filter to remove unwanted artefacts. A phase mask is created from the corrected phase image and the final SWI sequence is created by multiplying the magnitude images with the phase mask [13].
The final SWI sequence utilizes the susceptibility differences between adjacent tissues to produce tissue contrast and is extremely sensitive in the detection of diamagnetic and paramagnetic substances such as blood products, calcium, iron, free radicals from macrophage phagocytosis, and small vessels [13-16].

Although the final SWI sequence demonstrates low signal for both para- and diamagnetic substances, filtered phase sequences more accurately depict subtle differences in substance susceptibility $[14,15]$. On phase sequences paramagnetic substances, including free radicals, blood products, and iron, exhibit high signal, in contrast to diamagnetic substances such as calcium which return low signal [14-16].

In this case, MRI was performed on admission with subsequent MRI five months later. Initial SWI revealed hypointensity in the left lentiform nucleus only, with disproportionately more extensive hyperintensity on T1W MRI. Follow-up imaging five months later showed volume loss associated with improving hyperintensity on T1W MRI and more extensive and increased SWI hypointensity within the affected corpus striatum. The SWI sequence changes corresponded to areas of high signal on phase filtered SWI sequences. This suggests interval deposition of paramagnetic 
material, which could represent either mineralization or blood [13-15].

In our opinion, hemorrhage is unlikely based on the disproportionate extensive hyperintensity on the initial T1W sequence with comparatively little SWI hypointensity [1]. Interval progression of SWI hypointense signal within the left caudate nucleus also suggests an ongoing process of deposition rather than a single initial haemorrhagic event.

We postulate that the deposited paramagnetic material is most likely iron, accounting for the NECT head high attenuation. MRI imaging features further support this hypothesis, explaining the T1W MRI hyperintensity and SWI hypointensity and corresponding filtered phase SWI hyperintensity [17]. The progressive left corpus striatum malacic change demonstrated on follow-up imaging can be explained by iron-deposition-related neurotoxicity. The partial resolution of T1W hyperintensity at subsequent MRI with concomitant increased SWI hypointensity may reflect changes in the paramagnetic properties of iron during degradation, with resultant variable signal characteristics on T1W sequences.

\section{Summary}

Hemichorea hyperglycaemia is a rare syndrome often associated with hyperintensity on T1W imaging affecting the corpus striatum. We correlated this signal abnormality with the phase filtered SWI sequence to better define the nature of the corpus striatum signal change. We propose that deposition of paramagnetic material, in particular iron, is most likely responsible for the MRI signal abnormality associated with this condition.

\section{References}

[1] A. Cherian, B. Thomas, N. N. Baheti, T. Chemmanam, and C. Kesavadas, "Concepts and controversies in nonketotic hyperglycemia-induced hemichorea: further evidence from susceptibility-weighted MR imaging," Journal of Magnetic Resonance Imaging, vol. 29, no. 3, pp. 699-703, 2009.

[2] R. B. Dewey Jr. and J. Jankovic, "Hemiballism-hemichorea: clinical and pharmacologic findings in 21 patients," Archives of Neurology, vol. 46, no. 8, pp. 862-867, 1989.

[3] D. E. Shan, D. Ho, C. Chang, H. C. Pan, and M. M. H. Teng, "Hemichorea-hemiballism: an explanation for MR signal changes," American Journal of Neuroradiology, vol. 19, no. 5, pp. 863-870, 1998.

[4] A. N. Hegde, S. Mohan, N. Lath, and C. C. T. Lim, "Differential diagnosis for bilateral abnormalities of the basal ganglia and thalamus," Radiographics, vol. 31, no. 1, pp. 5-30, 2011.

[5] P. H. Lai, R. D. Tien, M. H. Chang et al., "Chorea-ballismus with nonketotic hyperglycemia in primary diabetes mellitus," American Journal of Neuroradiology, vol. 17, no. 6, pp. 1057-1064, 1996.

[6] M. Wintermark, N. J. Fischbein, P. Mukherjee, E. L. Yuh, and W. P. Dillon, "Unilateral putaminal CT, MR, and diffusion abnormalities secondary to nonketotic hyperglycemia in the setting of acute neurologic symptoms mimicking stroke," American Journal of Neuroradiology, vol. 25, no. 6, pp. 975-976, 2004.

[7] J. Nath, K. Jambhekar, C. Rao, and E. Armitano, "Radiological and pathological changes in hemiballism-hemichorea with striatal hyperintensity," Journal of Magnetic Resonance Imaging, vol. 23, no. 4, pp. 564-568, 2006.
[8] J. L. Hsu, H. C. Wang, and W. C. Hsu, "Hyperglycemia-induced unilateral basal ganglion lesions with and without hemichorea. A PET study," Journal of Neurology, vol. 251, no. 12, pp. 14861490,2004

[9] M. Fujioka, T. Taoka, Y. Matsuo, K. I. Hiramatsu, and T. Sakaki, "Novel brain ischemic change on MRI: delayed ischemic hyperintensity on T1-weighted images and selective neuronal death in the caudoputamen of rats after brief focal ischemia," Stroke, vol. 30, no. 5, pp. 1043-1046, 1999.

[10] S. Ohara, S. Nakagawa, K. Tabata, and T. Hashimoto, "Hemiballism with hyperglycemia and striatal T1-MRI hyperintensity: an autopsy report," Movement Disorders, vol. 16, no. 3, pp. 521-525, 2001.

[11] J. W. Neal, S. K. Singhrao, B. Jasani, and G. R. Newman, "Immunocytochemically detectable metallothionein is expressed by astrocytes in the ischaemic human brain," Neuropathology and Applied Neurobiology, vol. 22, no. 3, pp. 243-247, 1996.

[12] P. Mittal, "Hemichorea-hemiballism syndrome: a look through susceptibility weighted imaging," Annals of Indian Academy of Neurology, vol. 14, no. 2, pp. 124-126, 2011.

[13] V. Sehgal, Z. Delproposto, E. M. Haacke et al., "Clinical applications of neuroimaging with susceptibility-weighted imaging," Journal of Magnetic Resonance Imaging, vol. 22, no. 4, pp. 439450, 2005.

[14] R. J. Robinson and S. Bhuta, "Susceptibility-weighted imaging of the brain: current utility and potential applications," Journal of Neuroimaging, vol. 21, no. 4, pp. 189-204, 2011.

[15] B. Thomas, S. Somasundaram, K. Thamburaj et al., "Clinical applications of susceptibility weighted MR imaging of the brain-a pictorial review," Neuroradiology, vol. 50, no. 2, pp. 105-116, 2008.

[16] P. Lai, H. Chang, T. Chuang et al., "Susceptibility-weighted imaging in patients with pyogenic brain abscesses at 1. 5T: characteristics of the abscess capsule," American Journal of Neuroradiology, vol. 33, no. 5, pp. 910-914., 2012.

[17] M. Hernandez, L. Maconick, E. Tan, and J. Wardlaw, "Identification of mineral deposits in the brain on radiological images: a systematic review," European Radiology, vol. 22, no. 11, pp. 23712381, 2012. 


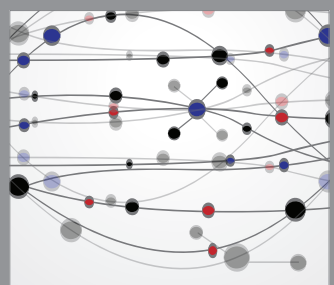

The Scientific World Journal
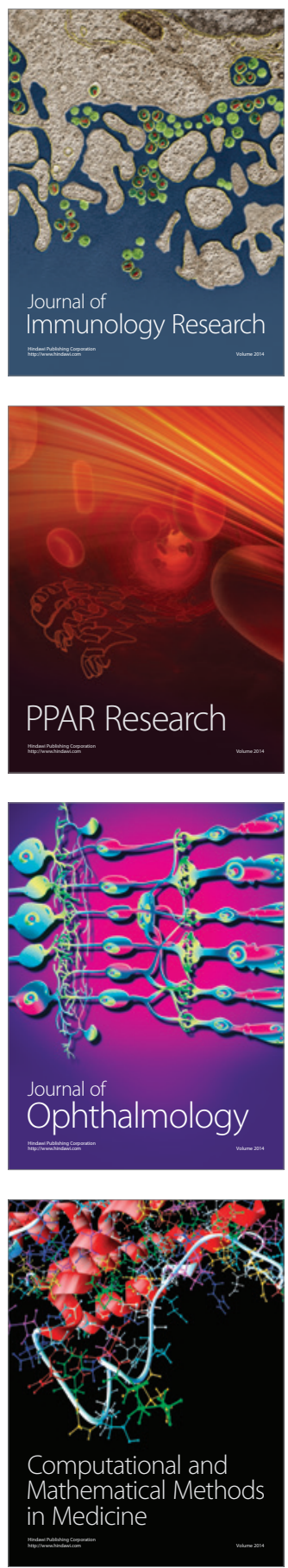

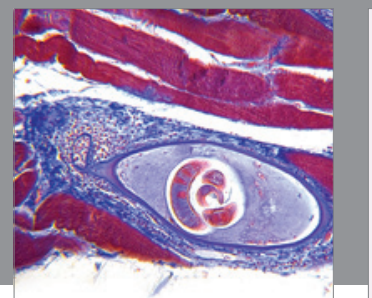

Gastroenterology

Research and Practice
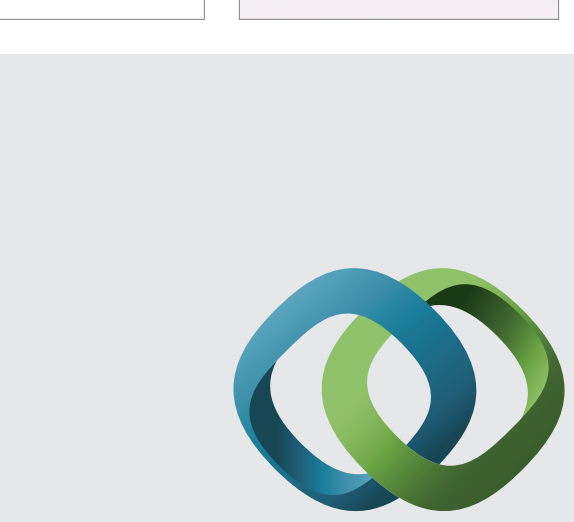

\section{Hindawi}

Submit your manuscripts at

http://www.hindawi.com
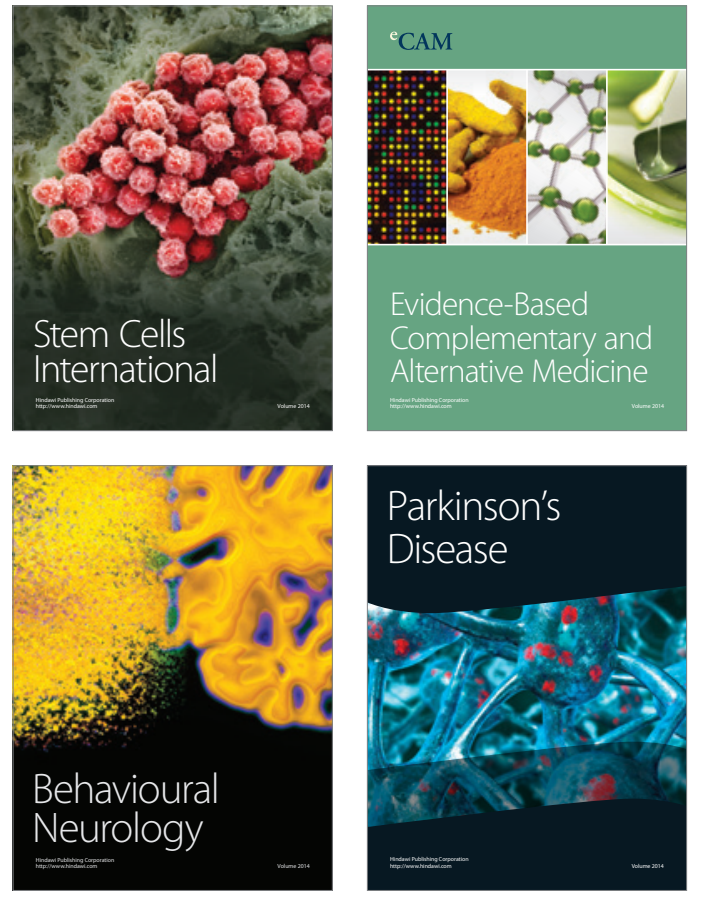
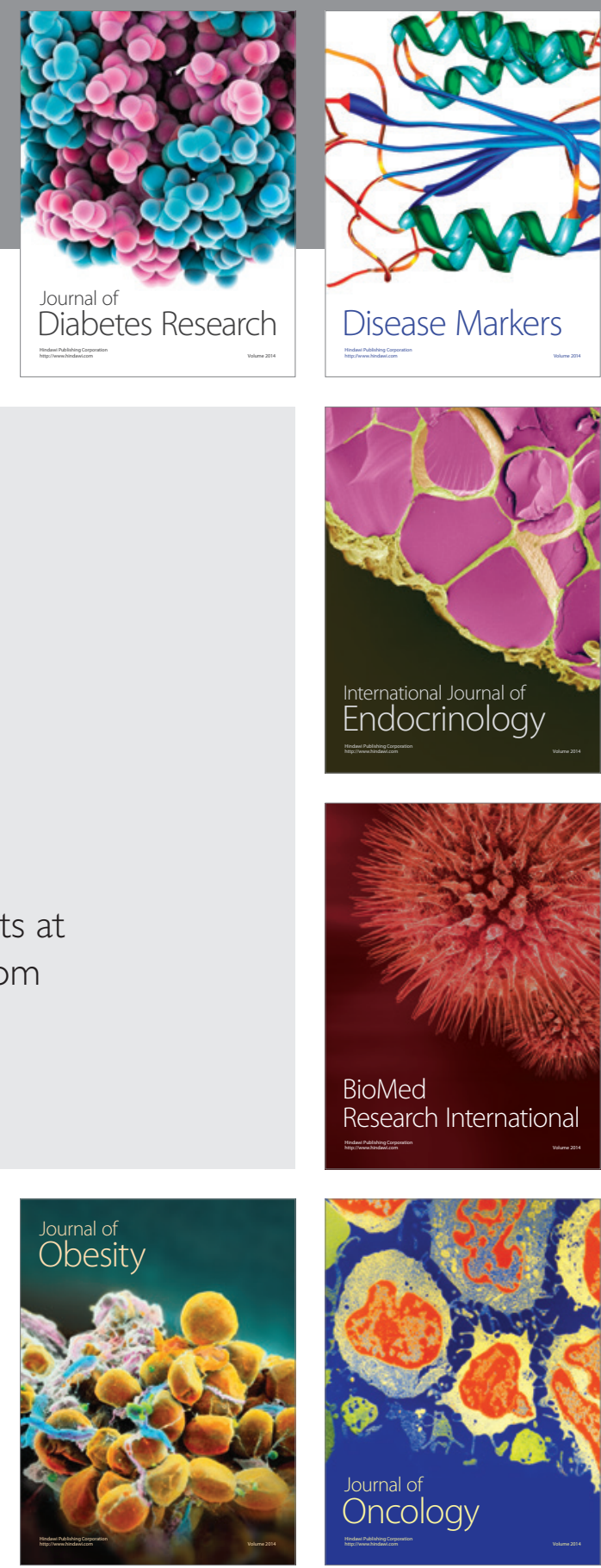

Disease Markers
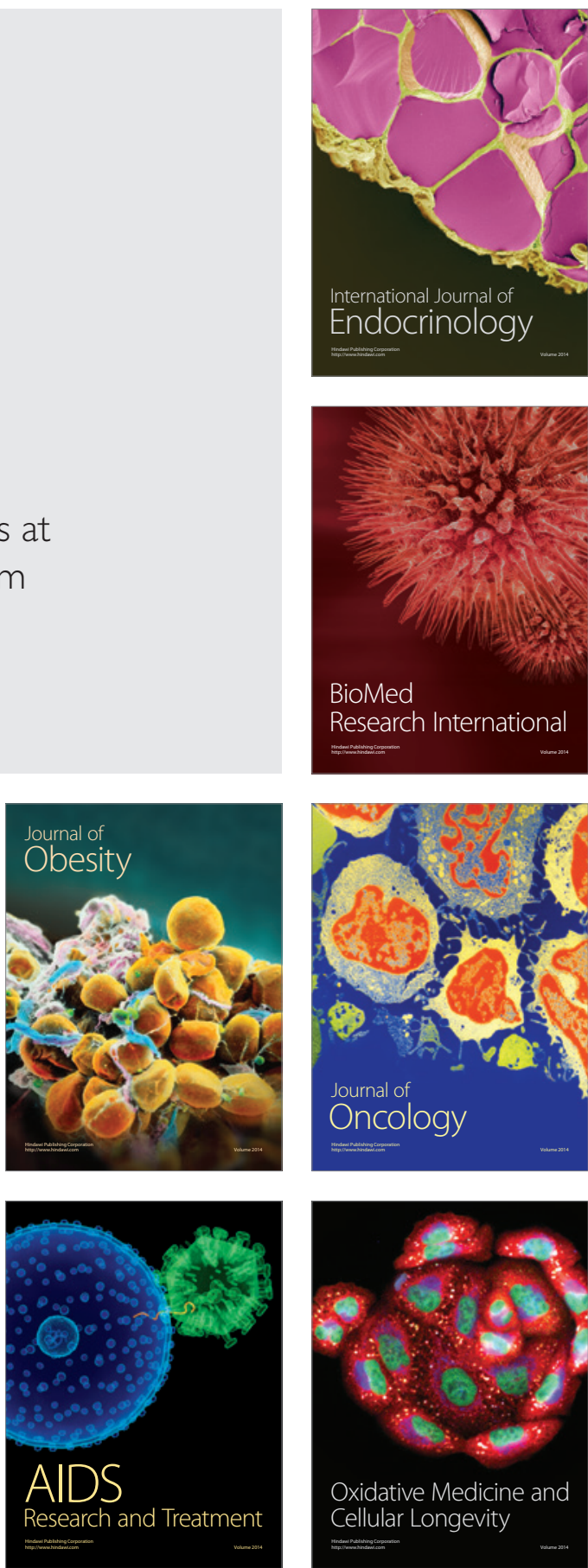